TRANSACTIONS OF THE

AMERICAN MATHEMATICAL SOCIETY

Volume 365, Number 5, May 2013, Pages 2447-2467

S 0002-9947(2012)05797-7

Article electronically published on October 9, 2012

\title{
CONVERGENCE AND BLOW-UP OF SOLUTIONS FOR A COMPLEX-VALUED HEAT EQUATION WITH A QUADRATIC NONLINEARITY
}

\author{
JONG-SHENQ GUO, HIROKAZU NINOMIYA, MASAHIKO SHIMOJO, \\ AND EIJI YANAGIDA
}

\begin{abstract}
This paper is concerned with the Cauchy problem for a system of parabolic equations which is derived from a complex-valued equation with a quadratic nonlinearity. First we show that if the convex hull of the image of initial data does not intersect the positive real axis, then the solution exists globally in time and converges to the trivial steady state. Next, on the onedimensional space, we provide some solutions with nontrivial imaginary parts that blow up simultaneously. Finally, we consider the case of asymptotically constant initial data and show that, depending on the limit, the solution blows up nonsimultaneously at space infinity or exists globally in time and converges to the trivial steady state.
\end{abstract}

\section{INTRODUCTION}

In this paper we consider the equation

$$
z_{t}=\Delta z+z^{2}
$$

where $z=z(x, t)$ is a complex-valued function of the spatial variable $x \in \mathbb{R}^{m}$ ( $m$ a positive integer) and the time $t \geq 0$, and $\Delta$ denotes the Laplace operator with respect to $x$. This equation appears as a one-dimensional model for the vorticity equation of incompressible and viscous fluids of three dimensions. If we write $z(x, t)=u(x, t)+i v(x, t)$, where $i=\sqrt{-1}$ and $u(x, t), v(x, t) \in \mathbb{R}$, then the equation above is rewritten as a system of parabolic equations

$$
\left\{\begin{array}{l}
u_{t}=\Delta u+u^{2}-v^{2}, \\
v_{t}=\Delta v+2 u v .
\end{array}\right.
$$

When $z$ is real-valued (i.e., $v \equiv 0$ ), then this system is reduced to the scalar equation

$$
u_{t}=\Delta u+u^{2} .
$$

This equation is a special case of the so-called Fujita equation ([9])

$$
u_{t}=\Delta u+u^{p}, \quad p>1,
$$

which has been studied extensively by many authors (see, e.g., [8, 10, 11, 12, 15. 17, 18, 19] and a recent monograph by Quittner and Souplet 25]). The aim of this paper is to make clear the difference of the dynamics of solutions between

Received by the editors August 30, 2011.

2010 Mathematics Subject Classification. Primary 35K57; Secondary 35K40, 35B44.

Key words and phrases. Complex-valued heat equation, parabolic system, convergence, blowup. 
the system (1.2) with $v \not \equiv 0$ and the scalar equation (1.3). For studies on other superlinear systems, we refer the reader to [1, 2, 4, 6, 14, 20, 21, 36.

Let us consider the initial value problem for (1.2) with an initial data

$$
(u(\cdot, 0), v(\cdot, 0))=\left(u_{0}, v_{0}\right) \in\left(L^{\infty}\left(\mathbb{R}^{m}\right) \cap C\left(\mathbb{R}^{m}\right)\right)^{2} .
$$

Then (1.2) has a unique solution $(u, v) \in\left(C\left([0, T) ; L^{\infty}\left(\mathbb{R}^{m}\right)\right)\right)^{2}$, where $T=T\left(u_{0}, v_{0}\right)$ $\in(0, \infty]$ denotes the maximal existence time of the solution. Moreover, we have either $T=\infty$, or

$$
T<\infty \quad \text { and } \quad \limsup _{t \rightarrow T}\left\{\|u(\cdot, t)\|_{L^{\infty}\left(\mathbb{R}^{m}\right)}+\|v(\cdot, t)\|_{L^{\infty}\left(\mathbb{R}^{m}\right)}\right\}=\infty .
$$

In the former case we say that the solution is global, while in the latter case we say that the solution blows up in a finite time. Also, $T$ is called the blow-up time of the solution if $T<\infty$. A point $x_{0} \in \mathbb{R}^{m}$ is said to be a blow-up point if there is a sequence $\left\{\left(x_{j}, t_{j}\right)\right\}$ such that $x_{j} \rightarrow x_{0}, t_{j} \uparrow T$ and $\left|u\left(x_{j}, t_{j}\right)\right|+\left|v\left(x_{j}, t_{j}\right)\right| \rightarrow \infty$ as $j \rightarrow \infty$. The set of all blow-up points is called the blow-up set. We investigate the global vs. nonglobal existence of solutions of (1.2).

Let us first consider a spatially homogeneous solution $(u, v)=(U(t), V(t))$. Then $(U(t), V(t))$ satisfies the following ODE system:

$$
\left\{\begin{array}{l}
U_{t}=U^{2}-V^{2}, \\
V_{t}=2 U V,
\end{array}\right.
$$

which can be solved explicitly as

$$
U(t)=\frac{T_{R}-t}{\left(T_{R}-t\right)^{2}+T_{I}^{2}}, \quad V(t)=\frac{T_{I}}{\left(T_{R}-t\right)^{2}+T_{I}^{2}},
$$

where

$$
T_{R}=\frac{U(0)}{U(0)^{2}+V(0)^{2}}, \quad T_{I}=\frac{V(0)}{U(0)^{2}+V(0)^{2}} .
$$

This implies that the solution on the positive $U$-axis is exceptional. Namely, the solution becomes unbounded at $t=T=T_{R}$ if $U(0)>0$ and $V(0)=0$, while $(U(t), V(t)) \rightarrow(0,0)$ as $t \rightarrow \infty$ otherwise. Thus we may expect that if a solution of (1.2) does not take any values on the positive $u$-axis, then the solution converges to the trivial steady state as $t \rightarrow \infty$. Indeed, the following result holds true:

Theorem 1.1. Suppose that the initial data satisfy

$$
u_{0}(x)-A v_{0}(x)<0 \quad \text { for all } x \in \mathbb{R}^{m}
$$

with some constant $A \in \mathbb{R}$. Then the solution of (1.2) with (1.4) exists globally in time and converges to $(0,0)$ as $t \rightarrow \infty$ uniformly in $\mathbb{R}^{m}$.

This theorem implies that even if $u_{0}$ is positive, there exist global solutions for (1.2) for any dimension $m \in \mathbb{N}$. On the other hand, it is known that all solutions of (1.3) with $u_{0}>0$ blow up if $m=1,2$. This is one of the differences between (1.2) and (1.3). See [9, 23] and the references in [25] for real-valued equations.

We also remark that the hypothesis of this theorem implies that the closure of the convex hull of $\operatorname{Im}(0)$ does not intersect the positive $u$-axis, where $\operatorname{Im}(t)$ represents the image of the solution on the $(u, v)$-plane defined by

$$
\operatorname{Im}(t):=\left\{(u(x, t), v(x, t)) \in \mathbb{R}^{2}: x \in \mathbb{R}^{m}\right\}, \quad t \geq 0 .
$$


As will be seen in the proof of Theorem 1.1, if the solution satisfies the condition (1.7), then $\operatorname{Im}(t)$ does not intersect the positive $u$-axis for all $t>0$.

When the hypothesis of Theorem 1.1 fails to hold, in view of the dynamics of the ODE system (1.5), it is not easy to see how the solution behaves. Therefore, it is interesting to ask whether or not the blow-up occurs if $v \not \equiv 0$. Also, for parabolic systems, it is interesting to determine whether all components blow up at the same time (which is called simultaneous blow-up) or only one component blows up and the other remains bounded. Such questions are considered in [24, 26, 34. Roughly speaking, their results claim that for a cooperative system, if the corresponding ODE system enjoys simultaneous or nonsimultaneous blow-up, adding the linear diffusion preserves the same phenomenon for the solution of a reaction-diffusion system also. See [25] for more references.

Let us consider the existence of blow-up solutions of our problem first.

Theorem 1.2. Let $m=1$. Suppose that

$$
\begin{aligned}
& \Theta(\omega):=\int_{\mathbb{R}}\left\{u_{0}(x) \cos (\omega x)+v_{0}(x) \sin (\omega x)\right\} d x>0, \\
& \int_{\mathbb{R}}\left\{v_{0}(x) \cos (\omega x)-u_{0}(x) \sin (\omega x)\right\} d x=0,
\end{aligned}
$$

for all $\omega \in \mathbb{R}$ and $\Theta(\omega)$ is continuous on $\mathbb{R}$. Then the solution of the Cauchy problem for (1.2) and (1.4) blows up in finite time.

Our equation has a strong relation with the viscous Constantin-Lax-Majda equation, which is a one-dimensional model for the vorticity equation. Let $\nu: \mathbb{R} \times$ $[0, T) \rightarrow \mathbb{R}$ be a solution of

$$
\nu_{t}=\nu_{x x}+\nu H \nu,
$$

where $\nu$ is a real-valued function and $H$ is the Hilbert transformation defined by

$$
[H f](x)=\frac{1}{\pi} \text { p.v. } \int_{-\infty}^{\infty} \frac{f(y)}{x-y} d y .
$$

We can see from the fundamental properties of the Hilbert transformation that the functions $\nu$ and $H \nu$ satisfy

$$
\nu_{t}=\nu_{x x}+\nu H \nu, \quad(H \nu)_{t}=(H \nu)_{x x}+\frac{1}{2}\left\{(H \nu)^{2}-\nu^{2}\right\} .
$$

Hence the function

$$
z:=\frac{1}{2 i}(\nu+i H \nu)
$$

satisfies $z_{t}=z_{x x}+z^{2}$. In [29, the author constructed the explicit solution of this problem which blows up in a finite time. It is also proved by [27, 28, that if Fourier coefficients of $\nu$ about Fourier sine series are all positive, then the function $\nu$ blows up in finite time. See also 22 for a generalization of the Constantin-Lax-Majda equation. The results in [27, 28] give us some examples of Theorem 1.2 for spatially periodic solutions. Here we provide more blow-up solutions without the restriction of periodicity.

The following theorem shows that the diffusion can induce simultaneous blow-up. This is not the case for an ODE system (1.5). Indeed, the only blow-up solution of the ODE system (1.5) is the positive solution, i.e., $U(t)>0$ and $V(t) \equiv 0$. 
Theorem 1.3. Let $(u, v)$ be a solution of the one-dimensional Cauchy problem for (1.2) with (1.4) such that $u(x, 0)=u(-x, 0)$ and $v(x, 0)=-v(-x, 0)$. Assume that $v_{0}(x)>0$ for all $x>0$. If the solution blows up in a finite time and its blow-up set is compact, then both components $u$ and $v$ blow up simultaneously.

About the compactness of blow-up sets, Friedman-Giga [7] considered the system $u_{t}=\Delta u+v^{q}, v_{t}=\Delta v+u^{p}$ with $p=q>1$ and constructed a radially symmetric solution that blows up only at the origin. A generalization of this result was recently obtained by Souplet 33 for $p, q>1$. It would be an interesting question to ask under what condition the blow-up set is compact.

Next, let us consider the case where the initial data are asymptotically constants. More precisely, we impose the following conditions on initial data:

$$
\begin{aligned}
& u_{0}, v_{0} \in C^{1}\left(\mathbb{R}^{m}\right), \quad 0 \leq u_{0} \leq M, u_{0} \not \equiv M, 0<v_{0} \leq L, \\
& \lim _{|x| \rightarrow \infty} u_{0}(x)=M, \lim _{|x| \rightarrow \infty} v_{0}(x)=N
\end{aligned}
$$

for some constants $L>0$ and $M>N \geq 0$.

The following theorem indicates that the solution of (1.2) with initial data satisfying the above conditions with $N>0$ behaves like the solution of (1.5) with $(U(0), V(0))=(M, N)$.

Theorem 1.4. Let $(u, v)$ be a solution of (1.2) with initial data $\left(u_{0}, v_{0}\right)$ satisfying (1.8) and (1.9). If $N>0$, then the solution of (1.2) with (1.4) exists globally in time and converges to $(0,0)$ as $t \rightarrow \infty$ uniformly in $\mathbb{R}^{m}$.

On the other hand, if the initial data satisfy the conditions (1.8) and (1.9) with $N=0$, then the solution blows up nonsimultaneously and blow-up occurs only at space infinity. More precisely, there are no (finite) blow-up points, but there exists a sequence $\left\{\left(x_{j}, t_{j}\right)\right\}$ such that $\left|x_{j}\right| \rightarrow \infty, t_{j} \uparrow T$ and $\left|u\left(x_{j}, t_{j}\right)\right|+\left|v\left(x_{j}, t_{j}\right)\right| \rightarrow \infty$ as $j \rightarrow \infty$.

Theorem 1.5. Let $(u, v)$ be a solution of (1.2) with initial data $\left(u_{0}, v_{0}\right)$ satisfying (1.8) and (1.9). If $N=0$, then the solution of (1.2) with (1.4) blows up at time $t=T(M)$ with $v \not \equiv 0$. More precisely, the component $u$ blows up only at space infinity and $v$ is bounded.

Note that the problem of blow-up at space infinity for scalar equations was considered in [13, 30, 31, and the corresponding cooperative system was analyzed in 32 .

This paper is organized as follows. In Section 2, we give a sufficient condition for the existence of global solutions and the convergence of solutions to the trivial steady state. In Section 3, we show the existence of solutions such that $u$ and $v$ blow up simultaneously in finite time. In Section 4, we consider a more general system than (1.2) with asymptotically constant initial data.

\section{Convergence to the trivial steady state}

In this section we give a proof of Theorem 1.1

Let $D(t)$ be a domain in $\mathbb{R}^{2}$ for each $t \geq 0$. We say that $\{D(t)\}_{t \geq 0}$ is (positively) invariant under the flow (1.5) if $(U(0), V(0)) \in D(0)$ implies $(U(t), V(t)) \in D(t)$ for all $t>0$. We need the following two properties related to the invariance (cf. Weinberger [37]). 
Lemma 2.1. Suppose that $D(t) \subset \mathbb{R}^{2}$ is convex for each $t \geq 0$ and $\{D(t)\}_{t \geq 0}$ is invariant under the flow (1.5). If $\operatorname{Im}\left(t_{0}\right) \subset D(0)$ for some $t_{0} \geq 0$, then $\operatorname{Im}(t) \subset D(t)$ for all $t>t_{0}$.

Lemma 2.2. Let $\left\{H_{j}\right\}_{1 \leq j \leq k}$ be a set of $C^{1}$ functions from $\mathbb{R}^{3}$ to $\mathbb{R}$. Suppose that $D(t)$ is expressed as

$$
D(t)=\bigcap_{j=1}^{k}\left\{(u, v) \in \mathbb{R}^{2}: H_{j}(u, v, t)<0\right\}, \quad t \geq 0 .
$$

Then $\{D(t)\}_{t \geq 0}$ is invariant under the flow (1.5) if

$$
\frac{d}{d t} H_{j}(U(t), V(t), t) \leq 0 \quad \text { on } \quad\left\{(u, v) \in \partial D: H_{j}(u, v, t)=0\right\}
$$

for all $j=1,2, \ldots, k$.

We introduce the polar coordinate system

$$
U(t)=r(t) \cos \theta(t), \quad V(t)=r(t) \sin \theta(t) .
$$

Then (1.5) is written as

$$
\begin{aligned}
& r_{t} \cos \theta-r \theta_{t} \sin \theta=r^{2}\left(\cos ^{2} \theta-\sin ^{2} \theta\right), \\
& r_{t} \sin \theta+r \theta_{t} \cos \theta=2 r^{2} \cos \theta \sin \theta,
\end{aligned}
$$

which yields

$$
r_{t}=r^{2} \cos \theta, \quad \theta_{t}=r \sin \theta .
$$

We first prove that $\operatorname{Im}(t)$ enters the left half-plane.

Proposition 2.3. Suppose that the hypothesis of Theorem 1.1 holds. Then the solution of (1.2) with (1.4) is uniformly bounded for $t \geq 0$, and there exist $t_{1}>0$ and $\pi / 2<\alpha<\pi<\beta<3 \pi / 2$ such that

$$
\operatorname{Im}\left(t_{1}\right) \subset\{(u, v)=(r \cos \theta, r \sin \theta): r>0, \alpha<\theta<\beta\} .
$$

Proof. We consider the case $A>0$ only, because the case $A \leq 0$ can be discussed in the same way.

For $R>0$, we define a bounded region by

$$
\begin{aligned}
D_{R}=\left\{(u, v) \in \mathbb{R}^{2}: u-A v<0, u^{2}+\left(v-\frac{R}{2}\right)^{2}\right. & <\frac{R^{2}}{4} \text { for } u \geq 0, \\
& \left.u^{2}+v^{2}<R^{2} \text { for } u<0\right\} .
\end{aligned}
$$

If $(U, V)$ is on the line $u=A v$, we have

$$
\frac{d}{d t}(U-A V)=U_{t}-A V_{t}=U^{2}-V^{2}-A U V=A^{2}\left(V^{2}-V^{2}-A V^{2}\right) \leq 0 .
$$

If $(U, V)$ is on the circle $u^{2}+(v-R / 2)^{2}=R^{2} / 4$, we have

$$
\begin{aligned}
\frac{d}{d t}\left\{U^{2}+\left(V-\frac{R}{2}\right)^{2}-\frac{R^{2}}{4}\right\} & =2 U U_{t}+2\left(V-\frac{R}{2}\right) V_{t} \\
& =2 U\left(U^{2}-V^{2}\right)+2\left(V-\frac{R}{2}\right)(2 U V) \\
& =2 U\left\{U^{2}+\left(V-\frac{R}{2}\right)^{2}-\frac{R^{2}}{4}\right\}=0 .
\end{aligned}
$$


Finally, if $(U, V)$ is on the circle $u^{2}+v^{2}=R^{2}$ and $U<0$, we have

$$
\begin{aligned}
\frac{d}{d t}\left(U^{2}+V^{2}-R^{2}\right) & =2 U U_{t}+2 V V_{t} \\
& =2 U\left(U^{2}-V^{2}\right)+2 V(2 U V) \\
& =2 U\left(U^{2}+V^{2}\right)<0 .
\end{aligned}
$$

Hence by Lemma 2.1, $D_{R}$ is invariant. Now if we take $R>0$ so large that $\operatorname{Im}(0)$ is contained in $D_{R}$, then $\operatorname{Im}(t)$ remains in $D_{R}$ for all $t>0$. Thus the solution is uniformly bounded for $t \geq 0$, and hence exists globally in time.

Set

$$
D_{1}(t):=\left\{(u, v) \in \mathbb{R}^{2}: u-a v+b(t)<0\right\},
$$

where $b(t)$ is a positive smooth function to be specified later. By assumption, we can take $a>0$ and $b(0)>0$ such that $\operatorname{Im}(0) \subset D_{1}(0)$. If $(U, V)$ is on the line $u-a v+b(t)=0$, we have

$$
\begin{aligned}
\frac{d}{d t}(U-a V+b) & =U_{t}-a V_{t}+b_{t} \\
& =U^{2}-V^{2}-a(2 U V)+b_{t} \\
& =(a V-b)^{2}-V^{2}-2 a(a V-b) V+b_{t} \\
& =-\left(a^{2}+1\right) V^{2}+b^{2}+b_{t} .
\end{aligned}
$$

Hence, from Lemma 2.2. $\left\{D_{1}(t)\right\}_{t \geq 0}$ is invariant if $b_{t} \leq-b^{2}$, which is satisfied by

$$
b(t)=\frac{b(0)}{b(0) t+1} .
$$

Next, we set

$$
D_{2}(t):=\left\{(u, v) \in \mathbb{R}^{2}: u \sin \omega(t)-v \cos \omega(t)<0\right\},
$$

where $\omega(t)$ is a smooth function satisfying $0<\omega(t)<\pi$ and $\omega(t)>\tan ^{-1}(1 / a)$. We note that $D_{1}(t) \cap D_{2}(t)$ is convex and two lines $\partial D_{1}(t)$ and $\partial D_{2}(t)$ intersect at a point, which is denoted by $(p(t), q(t))$. Clearly

$$
\sqrt{p^{2}(t)+q^{2}(t)} \geq \frac{1}{\sqrt{1+a^{2}}} b(t) .
$$

We shall show that $\left\{D_{1}(t) \cap D_{2}(t)\right\}_{t \geq 0}$ is invariant.

Since $\operatorname{Im}(0)$ is bounded, we can take $\omega(0) \in\left(\tan ^{-1}(1 / a), \pi / 2\right)$ appropriately so that $\operatorname{Im}(0) \subset D_{1}(0) \cap D_{2}(0)$. If $(U(t), V(t)) \in D_{1}(t) \cap \partial D_{2}(t)$, using polar coordinates, we have

$$
\begin{aligned}
\frac{d}{d t}\{U \sin \omega-V \cos \omega\}= & U_{t} \sin \omega+U \omega_{t} \cos \omega-V_{t} \cos \omega+V \omega_{t} \sin \omega \\
= & \left\{\left(r^{2} \cos ^{2} \theta-r^{2} \sin ^{2} \theta\right) \sin \omega+r \cos \theta \cdot \omega_{t} \cos \omega\right. \\
& \left.\quad-2 r^{2} \cos \theta \sin \theta \cos \omega+r \sin \theta \cdot \omega_{t} \sin \omega\right\}\left.\right|_{\theta=\omega} \\
= & -r^{2} \sin \omega+r \omega_{t} .
\end{aligned}
$$


Hence, from Lemma 2.2. $\left\{D_{1}(t) \cap D_{2}(t)\right\}_{t \geq 0}$ is invariant if $\omega_{t} \leq r \sin \omega$ on $D_{1}(t) \cap$ $\partial D_{2}(t)$. By (2.3), this condition is satisfied if

$$
\omega_{t}(t)=\frac{1}{1+a^{2}} b(t) \sin \omega(t) .
$$

Therefore, if $b(t)$ is given by (2.2), there is $t=t_{1}$ such that $\pi / 2<\omega\left(t_{1}\right)<\pi$. Since $\left\{D_{1}(t) \cap D_{2}(t)\right\}_{t \geq 0}$ is invariant, by using Lemma 2.1, we obtain

$$
\operatorname{Im}\left(t_{1}\right) \subset\left\{(u, v)=(r \cos \theta, r \sin \theta): r>0, \omega\left(t_{1}\right)<\theta<\pi+\tan ^{-1}(1 / a)\right\} .
$$

This completes the proof.

Next we prove that once $\operatorname{Im}(t)$ enters a left half-plane, then it remains in the left half-plane and approaches the origin.

Proposition 2.4. Suppose that

$$
\operatorname{Im}\left(t_{1}\right) \subset\{(u, v)=(r \cos \theta, r \sin \theta): 0<r<R, \alpha<\theta<\beta\}
$$

for some $t_{1}>0, R>0$ and $\pi / 2<\alpha<\pi<\beta<3 \pi / 2$. Then there exists a constant $C>0$ such that

$$
\operatorname{Im}(t) \subset\{(u, v)=(r \cos \theta, r \sin \theta): 0 \leq r \leq C / t, \alpha<\theta<\beta\}
$$

for all $t>t_{1}$.

Proof. Set

$$
D_{3}(t):=\{(u, v)=(r \cos \theta, r \sin \theta): 0 \leq r \leq \sigma(t), \alpha<\theta<\beta\} .
$$

By Lemma 2.2 again, (2.1) implies that $\left\{D_{3}(t)\right\}_{t \geq 0}$ is invariant if $\sigma_{t} \geq \sigma^{2} \max \{\cos \alpha$, $\cos \beta$. Solving this differential inequality with $\sigma\left(t_{1}\right)=R$, we obtain the conclusion by Lemma 2.1

Now Theorem 1.1 is proved as an immediate consequence of Propositions 2.3 and 2.4 .

Remark 2.5. Theorem 1.1 also holds true for the initial-boundary value problem under suitable boundary conditions. See Corollary on p. 50 or part (b) of Remarks on p. 45 in [35] for positively invariant regions.

\section{Simultaneous Blow-uP}

In this section, we consider the one-dimensional case $m=1$.

3.1. Proof of Theorem 1.2. First we shall show one criterion for blow-up. This is a simple generalization of the argument in [23] to our complex parabolic system. We define the Fourier transform of a measurable function $f \in L^{2}(\mathbb{R})$ by

$$
\hat{f}(\omega)=\frac{1}{\sqrt{2 \pi}} \int_{\mathbb{R}} f(x) e^{-i \omega \cdot x} d x
$$

and denote its inverse by

$$
f(x)=\frac{1}{\sqrt{2 \pi}} \int_{\mathbb{R}} \hat{f}(\omega) e^{i \omega \cdot x} d \omega .
$$

Then (1.1) is transformed into

$$
\hat{z}_{t}=-\omega^{2} \hat{z}+\hat{z} * \hat{z}
$$


where "*" stands for the convolution with respect to $\omega$ and $\hat{z}(\omega, t)$ is the Fourier transform of $z(x, t)$ with respect to the space variable $x$. The solution of (3.1) can be represented as

$$
\hat{z}(\omega, t)=e^{-\omega^{2} t} \hat{z}(\omega, 0)+\int_{0}^{t} e^{-\omega^{2}(t-s)}(\hat{z} * \hat{z})(\omega, s) d s .
$$

In the following, we always assume that $\hat{z}(\omega, t)$ is continuous.

The following lemma is essential in our argument.

Lemma 3.1. Suppose that $\hat{z}(\omega, 0)>0$ for any $\omega \in \mathbb{R}$. Then $\hat{z}(\omega, t)>0$ for all $\omega \in \mathbb{R}$ for $t>0$ as long as the solution of (3.1) exists.

Proof. To prove this lemma, we use the following iterations:

$$
\begin{aligned}
& \hat{z}_{1}(\omega, t)=\hat{z}(\omega, 0), \\
& \hat{z}_{n}(\omega, t)=e^{-\omega^{2} t} \hat{z}(\omega, 0)+\int_{0}^{t} e^{-\omega^{2}(t-s)}\left(\hat{z}_{n-1} * \hat{z}_{n-1}\right)(\omega, s) d s, n \geq 2
\end{aligned}
$$

Then the lemma follows easily by an iterated argument.

Let $z_{0}:=u_{0}+i v_{0}$ and $\hat{z}_{0}$ be its Fourier transform. Theorem 1.2 is a consequence of the following theorem, since the conditions in Theorem 1.2 imply that $\hat{z}_{0}=$ $\Theta / \sqrt{2 \pi}$ is positive and continuous.

Theorem 3.2. Let $(u, v)$ be a solution of (1.2) with (1.4) on $\mathbb{R}$. Assume that $\hat{z}_{0}(\omega)$ is positive and continuous in $\omega \in \mathbb{R}$. Then the solution $(u, v)$ blows up in a finite time.

Proof. We prove this theorem by contradiction. Assume that the solution exists for all $t>0$. By the continuity of $\hat{z}_{0}$ at $\omega=0$, there is a positive constant $\delta$ such that

$$
\min _{|\omega| \leq \delta / 2} \hat{z}_{0}(\omega)>8 \delta
$$

Choose a constant $\tau$ with $\tau \geq \ln 2 / \delta^{2}$. Set $B=[-\delta / 2, \delta / 2]$ and divide $B$ into $N$ intervals, namely,

$$
B_{j}=\left[-\frac{\delta}{2}+\frac{(j-1)) \delta}{N},-\frac{\delta}{2}+\frac{j \delta}{N}\right], \quad j=1,2, \ldots, N .
$$

Next, we define the local mean value and its minimum by

$$
M_{j}(t)=M_{j}(t ; \delta, N):=\frac{1}{\left|B_{j}\right|} \int_{\omega \in B_{j}} \hat{z}(\omega, t) d \omega, \quad M(t)=M(t ; \delta, N):=\min _{1 \leq j \leq N} M_{j}(t) .
$$

Using Lemma 3.1 and Lebesgue's Differentiation Theorem, we may assume that

$$
\hat{z}(\omega, t) \geq \frac{M(t)}{2} \text { for } \omega \in B, 0 \leq t \leq \tau,
$$

by choosing $N$ sufficiently large. Thus, by differentiating the function $M_{j}(t)$ and using (3.1) and (3.3), we obtain the following differential inequalities:

$$
M_{j}^{\prime}(t) \geq-\delta^{2} M_{j}(t)+\frac{\delta}{4} M^{2}(t), \quad j=1,2, \ldots, N .
$$

Hence $M_{j}(t)$ satisfies the integral inequality

$$
e^{\delta^{2} t} M_{j}(t) \geq M_{j}(0)+\frac{\delta}{4} \int_{0}^{t} e^{\delta^{2} s} M^{2}(s) d s \geq M(0)+\frac{\delta}{4} \int_{0}^{t} e^{\delta^{2} s} M^{2}(s) d s
$$


for each $j$. Since $M(t)=M_{j}(t)$ for some $j$, we conclude that

$$
e^{\delta^{2} t} M(t) \geq M(0)+\frac{\delta}{4} \int_{0}^{t} e^{\delta^{2} s} M^{2}(s) d s \quad \text { for } \quad 0 \leq t \leq \tau .
$$

This implies that $M(t)$ blows up before the time $\tau$, since $M(0)>4 \delta$. Then by taking $x=0$ in the inverse Fourier transform we conclude that the function $u(0, t)$ of the solution $(u, v)$ of (1.2) blows up in a finite time.

Remark 3.3. Under the assumption of Theorem 3.2, we see that

$$
\begin{aligned}
& u(x, t)=\frac{1}{\sqrt{2 \pi}} \int_{\mathbb{R}} \hat{z}(\omega, t) \cos \omega x d \omega, \\
& v(x, t)=\frac{1}{\sqrt{2 \pi}} \int_{\mathbb{R}} \hat{z}(\omega, t) \sin \omega x d \omega,
\end{aligned}
$$

and that $u$ is an even function and $v$ is an odd one with respect to $x$, in particular,

$$
u_{0}(x)=u_{0}(-x), \quad v_{0}(x)=-v_{0}(-x) \quad \text { for } x \in \mathbb{R} .
$$

Conversely if $\left(u_{0}, v_{0}\right)$ satisfies (3.4), then $\hat{z}_{0}$ is real. A typical example of such initial data is

$$
u_{0}(x)=\left(3-4 x^{2}\right) e^{-x^{2}}, \quad v_{0}(x)=2 x e^{-x^{2}}, \quad \hat{z}_{0}(\omega)=\frac{1+\omega+\omega^{2}}{\sqrt{2}} e^{-\omega^{2} / 4} .
$$

3.2. Simultaneous blow-up for one-dimensional case. In this subsection we shall give a proof of Theorem 1.3. Let $(u, v)$ be a solution of the Cauchy problem for (1.2) with (1.4) satisfying (3.4). Then we have $u(x, t)=u(-x, t)$ and $v(x, t)=$ $-v(-x, t)$, and especially, $u_{x}(0, t)=0$ and $v(0, t)=0$ for all $t \in(0, T)$. Moreover, we assume that

$$
v_{0}(x)>0 \text { for } x>0 .
$$

In order to exclude the blow-up at space infinity, we also assume the following assumption:

(H) Blow-up set of the solution $(u, v)$ is compact.

We first introduce a rescaling of variables to analyze the solution near a blow-up point. For any $\eta \in \mathbb{R}$ and $T>0$, we define $W=W_{\eta, T}:=W(\xi, s ; \eta, T)$ by

$$
W(\xi, s ; \eta, T):=(T-t) u(x, t), \quad \xi:=\frac{x-\eta}{\sqrt{T-t}}, \quad s:=-\ln (T-t)
$$

for $\xi \in \mathbb{R}$ and $s>s_{0}:=-\ln T$. Then $W$ satisfies

$W_{s}=W_{\xi \xi}-\frac{\xi}{2} W_{\xi}-W+W^{2}+g, \quad$ where $\quad g(y, s):=-e^{-2 s} v^{2}\left(\eta+e^{-s / 2} \xi, T-e^{-s}\right)$ and $W\left(\xi, s_{0}\right)=T u_{0}(\eta+\xi \sqrt{T})$. Now we introduce the energy functional

$$
E[\varphi]=\int_{\mathbb{R}}\left(\frac{\varphi_{\xi}^{2}}{2}+\frac{\varphi^{2}}{2}-\frac{\varphi^{3}}{3}\right) \rho d \xi, \quad \varphi \in H_{\rho}^{1} \cap L_{\rho}^{3},
$$

where $\rho(\xi):=(4 \pi)^{-1 / 2} e^{-\xi^{2} / 4}$ and

$$
\begin{gathered}
L_{\rho}^{q}=L_{\rho}^{q}(\mathbb{R}):=\left\{\varphi \in L^{q}(\mathbb{R}): \int_{\mathbb{R}}|\varphi|^{q} \rho d y<\infty\right\}, \\
H_{\rho}^{1}=H_{\rho}^{1}(\mathbb{R}):=\left\{\varphi \in L_{\rho}^{2}(\mathbb{R}): \varphi_{\xi} \in L_{\rho}^{2}(\mathbb{R})\right\}
\end{gathered}
$$


for $q \geq 1$. In the following, we simply write $W(s):=W(\cdot, s)$ and $g(s):=g(\cdot, s)$, and so on. Now we set

$$
F[g](s):=\frac{1}{2} \int_{s_{0}}^{s} \int_{\mathbb{R}} g^{2}(\xi, \tau) \rho d \xi d \tau, \quad J[W(s)]:=E[W(s)]-F[g](s) .
$$

If $v$ is bounded for $0 \leq t \leq T$, then there is a positive constant $K_{1}$ satisfying

$$
|F[g](s)| \leq K_{1}, \quad s>s_{0} .
$$

By a simple calculation, we obtain

$\frac{d}{d s} J[W(s)]=-\int_{\mathbb{R}} W_{s}^{2}(s) \rho d \xi+\int_{\mathbb{R}} g(s) W_{s}(s) \rho d \xi-\frac{1}{2} \int_{\mathbb{R}} g^{2}(s) \rho d \xi \leq-\frac{1}{2} \int_{\mathbb{R}} W_{s}^{2}(s) \rho d \xi$ for any $s>s_{0}$. This means that $J[W(s)]$ is monotone decreasing in $s>s_{0}$ and that

$$
E[W(s)]<J[W(0)]+K_{1}:=K_{2}, \quad s>s_{0},
$$

if $v$ is bounded for $0 \leq t \leq T$.

The local convergence result can be proved by the standard argument similar to [10, 11, 12.

Proposition 3.4. Let $(u, v)$ be a solution of (1.2) with (1.4) on $\mathbb{R}$ that blows up at $t=T \in(0, \infty)$. Assume that $v$ is uniformly bounded in $\mathbb{R} \times[0, T)$. Then $u$ satisfies

$$
\lim _{t \rightarrow T}(T-t) u\left(a+(T-t)^{1 / 2} \xi, t\right)=1
$$

uniformly on $|\xi| \leq C$ for any $C>0$.

Although the proof of this proposition is quite similar to that of Section 6.A of [12] with a small modification, for the reader's convenience we shall give a proof here.

Proof. First, let us show that $u$ is bounded from below. The assumption of the uniform boundedness of $v$ implies that there is a constant $M>0$ such that $|v(x, t)| \leq M$ for all $x \in \mathbb{R}$ and $t \in[0, T)$. Hence $u$ satisfies a differential inequality $u_{t} \geq u_{x x}+u^{2}-M^{2}$. Thus by a simple comparison principle, we obtain an inequality: $u(x, t) \geq \inf _{x \in \mathbb{R}}\left\{u_{0}(x)\right\}-M^{2} t$. Hence $u$ is uniformly bounded from below.

Next, we show that there exist constants $K_{i}(i=3,4,5)$ and $s_{*}>-\ln T$ such that

$$
\begin{aligned}
& \int_{s_{*}}^{\infty} \int_{\mathbb{R}}\left|W_{s}\right|^{2} \rho d \xi d s \leq K_{3}, \\
& \int_{\mathbb{R}}|W(s)|^{2} \rho d \xi \leq K_{4}, \\
& \int_{s}^{s+1}\left(\int_{\mathbb{R}}|W|^{3} \rho d \xi\right)^{2} d s \leq K_{5}
\end{aligned}
$$

for all $s \geq s_{*}$ for some $s_{*}>s_{0}$.

In the following, for any measurable function $f(x)$, we define $f_{-}(x)$ by $f_{-}(x)=$ $-f(x)$ when $f(x)<0$, and $f_{-}(x)=0$ otherwise. Note that $W_{-}$converges to zero 
as $s \rightarrow \infty$, due to the fact that $u$ is bounded from below. Since $W=|W|-2 W_{-}$, we have

$$
\begin{aligned}
\frac{1}{2} & \frac{d}{d s} \int_{\mathbb{R}} W^{2}(s) \rho d \xi \\
& =\int_{\mathbb{R}}\left(-W_{\xi}^{2}(s)-W^{2}(s)+W^{3}(s)+g(s) W(s)\right) \rho d \xi \\
& =-2 E[W(s)]+\frac{1}{3} \int_{\mathbb{R}} W^{3}(s) \rho d \xi+\int_{\mathbb{R}} g(s) W(s) \rho d \xi \\
& =-2 J[W(s)]-2 F[g](s)+\frac{1}{3} \int_{\mathbb{R}} W^{3}(s) \rho d \xi+\int_{\mathbb{R}} g(s) W(s) \rho d \xi \\
& \geq-2 J[W(s)]-K_{6}+\frac{1}{3} \int_{\mathbb{R}}|W(s)|^{3} \rho d \xi-\frac{2}{3} \int_{\mathbb{R}} W_{-}^{3}(s) \rho d \xi-\frac{1}{2} \int_{\mathbb{R}} W^{2}(s) \rho d \xi
\end{aligned}
$$

The lower bound of $u$ and the definition of $\rho$ imply that

$$
\frac{2}{3} \int_{\mathbb{R}} W_{-}^{3}(s) \rho d \xi \leq K_{7}
$$

with some positive constant $K_{7}$. Then we have

$$
\frac{1}{2} \frac{d}{d s} \int_{\mathbb{R}} W^{2}(s) \rho d \xi \geq-2 J[W(s)]-K_{6}-K_{7}+\frac{1}{3}\left(\int_{\mathbb{R}} W^{2}(s) \rho d \xi\right)^{\frac{3}{2}}-\frac{1}{2} \int_{\mathbb{R}} W^{2}(s) \rho d \xi
$$

for some constant $K_{7}$. Since $W$ is defined for all $s \geq s_{0}$, the right-hand side of the above inequality cannot be positive for all $s$. We obtain an $L^{2}$-bound (3.7) for all $s \geq s_{*}$ for some $s_{*}>s_{0}$. Combining the monotonicity and (3.9), we also get a lower bound of $J[W(s)]$, which also yields a lower bound of $E[W(s)]$; namely, there is a constant $K_{8}$ such that

$$
E[W(s)] \geq-K_{8} \quad \text { for all } s \geq s_{*} .
$$

Furthermore, by using (3.7) and an upper bound for $E[W(s)]$, we obtain

$$
\begin{aligned}
\frac{1}{3} \int_{\mathbb{R}}|W(s)|^{3} \rho d \xi= & \frac{2}{3} \int_{\mathbb{R}} W_{-}^{3}(s) \rho d \xi+\frac{1}{3} \int_{\mathbb{R}} W^{3}(s) \rho d \xi \\
\leq & K_{7}+\int_{\mathbb{R}} W(s) W_{s}(s) \rho d \xi+2 E[W(s)]-\int_{\mathbb{R}} g(s) W(s) \rho d \xi \\
\leq & K_{7}+\int_{\mathbb{R}} W(s) W_{s}(s) \rho d \xi+2 K_{2}+\frac{1}{2} \int_{\mathbb{R}} g^{2}(s) \rho d \xi \\
& +\frac{1}{2} \int_{\mathbb{R}} W^{2}(s) \rho d \xi \\
\leq & K_{7}+\frac{1}{2} \int_{\mathbb{R}} W_{s}^{2}(s) \rho d \xi+2 K_{2}+\frac{1}{2} \int_{\mathbb{R}} g^{2}(s) \rho d \xi+\int_{\mathbb{R}} W^{2}(s) \rho d \xi \\
\leq & K_{9}+\frac{1}{2} \int_{\mathbb{R}} W_{s}^{2}(s) \rho d \xi \leq K_{9}-\frac{d}{d s} E[W(s)]+K_{10} e^{-4 s}
\end{aligned}
$$

with some positive constants $K_{9}$ and $K_{10}$. Hence we have (3.8). Combining (3.5) and the bound for $E[W(s)]$ gives us (3.6).

Now, we rewrite the equation for $W$ as

$$
W_{s}=W_{\xi \xi}-\frac{\xi}{2} W_{\xi}+(W-1) W-e^{-2 s} v^{2} .
$$


Using

$$
|W-1|^{3} \leq|W|^{3}+3|W|^{2}+3|W|+1
$$

and (3.8), we can easily show that

$$
\int_{s}^{s+1}\left(\int_{|\xi| \leq 1}|W-1|^{3} \rho d \xi\right)^{2} d s \leq K_{11}, \quad s \geq s_{*} .
$$

Applying this estimate, (3.7) and Theorem 8.1 in Chapter III of [16 to (3.10) yield an $L^{\infty}$-bound:

$$
\left|W_{\eta, T}(\xi, s)\right| \leq K_{12} \quad \text { for } \quad|\xi| \leq \frac{1}{2}, s \geq s_{*}+\delta
$$

for any $\delta>0$. The relation $W_{\eta, T}(\xi, s)=W_{0, T}\left(\xi+e^{\frac{s}{2}} \eta, s\right)$ gives us a uniform bound of $\left|W_{\eta, T}(\xi, s)\right|$ for all $|\xi| \leq c$ and $s \geq s^{*}+\delta$, where $c>0$ is any positive constant. By (3.6) we conclude that the $\omega$-limit set of $W_{\eta, T}$ is a compact connected set contained in the set of bounded solutions $\Phi=\Phi(\xi)$ of the problem

$$
\Phi_{\xi \xi}-\frac{\xi}{2} \Phi_{\xi}-\Phi+\Phi^{2}=0, \quad \xi \geq 0, \quad \Phi_{\xi}(0)=0 .
$$

It can be easily seen from the proof of Theorem 1 in [10] that the only bounded solution of this one-dimensional elliptic problem is a constant solution. Thus the $\omega$-limit set of $W_{\eta, T}$ is contained in the set $\{1,0\}$. Furthermore, for a blow-up point $\eta=a$, the possibility 0 is excluded from the $\omega$-limit set in view of Theorem 2.1 of [12, since $u$ satisfies the inequality $\left|u_{t}-\Delta u\right| \leq K^{\prime}\left(1+u^{2}\right)$ for some constant $K^{\prime}>0$ near $x=a$. This completes the proof.

Note that $v$ is bounded for a nonsimultaneous blow-up solution $(u, v)$ of (1.2). From the above proof, we have the following corollary.

Corollary 3.5. Let $(u, v)$ be a nonsimultaneous blow-up solution of (1.2) with (1.4) on $\mathbb{R}$ that blows up at $t=T \in(0, \infty)$. Then $u$ is uniformly bounded from below.

Now, we prove that the simultaneous blow-up does occur under certain conditions.

Proof of Theorem 1.3. We shall assume that $v$ is uniformly bounded for all $x \in \mathbb{R}$ and $t \in[0, T)$ to get a contradiction. By the assumption that the blow-up set is compact and the symmetry, we only need to consider two cases for the blow-up point $a \geq 0$ of $u$. One is when $a>0$ and the other case is $a=0$.

First, we consider the case when $a>0$. At any blow-up point $a>0$ of $u$, there exist $c>0$ and $\delta \in[0, a / 2]$ such that $v(x, t) \geq c$ for all $(x, t) \in[a-\delta, a+\delta] \times[0, T]$, since $v$ is positive for $x>0$ by the strong maximum principle.

For any fixed $\theta \in(0,1 / 4)$, choosing $R \gg 1$ so that $\pi^{2} /\left(4 R^{2}\right)<1 / 2$, by Proposition 3.4 there exists $t_{0} \in(0, T)$ sufficiently close to $T$ such that

$$
u(x, t) \geq \frac{1-\theta}{T-t} \quad \text { for } \quad|x-a| \leq R \sqrt{T-t}, t \in\left[t_{0}, T\right) .
$$

Here we may also assume that $T-t_{0} \ll 1$ so that $a \geq 2 R \sqrt{T-t_{0}}$. 
Now, let us consider a rescaling at $x=a, t=T$ to introduce $W=W_{a, T}:=$ $W(\xi, s ; a, T)$ and $Z=Z_{a, T}:=Z(\xi, s ; a, T)$ by

$$
\begin{gathered}
W(\xi, s ; a, T):=(T-t) u(x, t), \quad Z(\xi, s ; a, T):=(T-t) v(x, t), \\
\xi=\frac{x-a}{\sqrt{T-t}}, \quad s=-\ln (T-t)
\end{gathered}
$$

for $\xi \in \mathbb{R}$ and $s>s_{0}:=-\ln T$. Then $(W, Z)$ satisfies

$$
\left\{\begin{array}{l}
W_{s}=W_{\xi \xi}-\frac{\xi}{2} W_{\xi}-W+W^{2}-Z^{2} \\
Z_{s}=Z_{\xi \xi}-\frac{\xi}{2} Z_{\xi}-Z+2 W Z
\end{array}\right.
$$

Then we have from 3.11 the inequality

$$
Z_{s} \geq Z_{\xi \xi}-\frac{\xi}{2} Z_{\xi}+(1-2 \theta) Z
$$

for all $(\xi, s) \in(-R, R) \times\left(-\ln \left(T-t_{0}\right), \infty\right)$. Here we have used the fact that $Z>0$ for $\xi \in(-a / \sqrt{T-t}, \infty)$, for any $t>0$, and the fact that $a / \sqrt{T-t}>R$ for all $t \geq t_{0}$.

Let us define $\psi(\xi)=\psi_{1, D}^{R}(\xi)=\cos [\pi \xi /(2 R)]$. Then we have

$$
\psi_{\xi \xi}-\frac{\xi}{2} \psi_{\xi}+(1-2 \theta) \psi=\left[1-2 \theta-\pi^{2} /\left(4 R^{2}\right)\right] \psi-\frac{\xi}{2} \psi_{\xi} \geq 0 \quad \text { for } \xi \in(-R, R) .
$$

By choosing sufficiently small $\varepsilon>0$, we may assume $Z\left(\xi,-\ln \left(T-t_{0}\right)\right) \geq \varepsilon \psi(\xi)$ for all $|\xi| \leq R$. Here we used the positivity of $v\left(\cdot, t_{0}\right)$ on $\left\{x \in \mathbb{R}:|x-a| \leq R \sqrt{T-t_{0}}\right\}$. Now, we can apply the comparison principle to conclude that $Z(\xi, s) \geq \varepsilon \psi(\xi)$ for all $\xi$ satisfying $|\xi| \leq R$ as long as $s \geq-\ln \left(T-t_{0}\right)$. This means that $v(x, t) \geq$ $\varepsilon(T-t)^{-1} \psi(|x-a| / \sqrt{T-t})$ for all $|x-a| \leq R \sqrt{T-t}$ and $t \geq t_{0}$. Hence we conclude that $v(a, t) \rightarrow \infty$ as $t \rightarrow T$. Thus we get a contradiction.

Next, we consider the second case when $a=0$. Let $\lambda(R)$ denote the first eigenvalue of the Laplace operator on the three-dimensional ball of radius $R>0$ centered at the origin under the zero Dirichlet boundary condition. Note that $\lambda(R)=R^{-2} \lambda(1)$. By Proposition 3.4. for a given $\theta \in(0,1 / 4)$ and $R>0$ with $\lambda(R)<1 / 2$, there exists $t_{0} \in(0, T)$ sufficiently close to $T$ such that

$$
u(r, t) \geq \frac{1-\theta}{T-t}, \quad r \leq R \sqrt{T-t}, t \in\left[t_{0}, T\right) .
$$

When $a=0$, we consider the transformation $\mu=v / r$, where $r=|x|$. Then (1.2) becomes

$$
u_{t}=u_{r r}+u^{2}-r^{2} \mu^{2}, \quad \mu_{t}=\mu_{r r}+\frac{2}{r} \mu_{r}+2 u \mu
$$

with the zero Neumann boundary conditions for $u$ and $\mu$ at the origin, due to the symmetry assumption.

Again, we consider a rescaling $W=W_{0, T}:=W(\xi, s ; 0, T)$ and $Y=Y_{0, T}:=$ $Y(\xi, s ; 0, T)$ by

$$
\begin{aligned}
& W(\xi, s ; 0, T):=(T-t) u(r, t), \quad Y(\xi, s ; 0, T):=(T-t) \mu(r, t), \\
& \xi=\frac{r}{\sqrt{T-t}}, \quad s=-\ln (T-t)
\end{aligned}
$$


for $\xi \in \mathbb{R}$ and $s>s_{0}:=-\ln T$. Then $(W, Y)$ satisfies

$$
\left\{\begin{array}{c}
W_{s}=W_{\xi \xi}-\frac{\xi}{2} W_{\xi}-W+W^{2}-Z^{2} \\
Y_{s}=Y_{\xi \xi}+\frac{2}{\xi} Y_{\xi}-\frac{\xi}{2} Y_{\xi}-Y+2 W Y
\end{array}\right.
$$

and

$$
Y_{\xi}(0, s)=0 \quad \text { for } s>s_{0}, \quad W\left(\xi, s_{0}\right)=T u_{0}(\xi \sqrt{T}), \quad Y\left(\xi, s_{0}\right)=\sqrt{T} \xi^{-1} v_{0}(\xi \sqrt{T}) .
$$

Now we start the same argument as in the case when $a>0$ for a radially symmetric extended function $\mu$ defined on $\mathbb{R}^{3}$. Consider the corresponding positive eigenfunction $\varphi(\xi)=\varphi_{1, D}^{R}(\xi)$ of the Laplace operator on the three-dimensional ball of radius $R$ centered at the origin under the zero Dirichlet boundary condition. Then $\varphi$ satisfies

$$
\varphi_{\xi \xi}+\frac{2}{\xi} \varphi_{\xi}-\frac{\xi}{2} \varphi_{\xi}+(1-2 \theta) \varphi=\{1-2 \theta-\lambda(R)\} \varphi-\frac{\xi}{2} \varphi_{\xi} \geq 0
$$

for all $0<\xi \leq R$, since $\varphi$ is positive and monotone decreasing. Without loss of generality, we assume that the maximum of $\varphi(\xi)$ is equal to 1. Choosing sufficiently small $\varepsilon>0$, we may assume that $Y\left(\xi,-\ln \left(T-t_{0}\right)\right) \geq \varepsilon \varphi(\xi)$ for all $0 \leq \xi \leq R$. Here we use the positivity of $\mu\left(r, t_{0}\right)$ for $0 \leq r \leq R \sqrt{T-t_{0}}$, which follows from the strong maximum principle. Again, we can apply the comparison principle to conclude that $Y(\xi, s) \geq \varepsilon \varphi(\xi)$ for all $0 \leq \xi \leq R$ and $s \geq-\ln \left(T-t_{0}\right)$. This yields that $r^{-1} v(r, t) \geq \varepsilon(T-t)^{-1} \varphi(r / \sqrt{T-t})$ for all $0 \leq r \leq R \sqrt{T-t}$ and $t \geq t_{0}$. This inequality can be written as $v(r, t) \geq \varepsilon(T-t)^{-1} r \varphi(r / \sqrt{T-t})$. By substituting $r=\sqrt{T-t}$ and letting $t \rightarrow T$, we conclude that $v$ blows up and get a contradiction. This completes the proof.

\section{Asymptotically constant initial data}

4.1. General system. In this subsection we consider the following more general system than (1.2):

$$
\begin{cases}\mathbf{u}_{t}=D \Delta \mathbf{u}+\mathbf{f}(\mathbf{u}), & x \in \mathbb{R}^{m}, t>0, \\ \mathbf{u}(x, 0)=\mathbf{u}_{0}(x), & x \in \mathbb{R}^{m},\end{cases}
$$

where $\mathbf{u}(x, t)=\left(u_{1}(x, t), \ldots, u_{k}(x, t)\right) \in \mathbb{R}^{k}, \mathbf{f}=\left(f_{1}, \ldots, f_{k}\right)$ is a smooth mapping from $\mathbb{R}^{k}$ to $\mathbb{R}^{k}, D=\operatorname{diag}\left(d_{1}, \ldots, d_{k}\right)$ with $d_{j}>0$ for $j=1,2, \ldots, k$. We assume that $\mathbf{u}_{0} \in\left(L^{\infty}\left(\mathbb{R}^{m}\right) \cap C\left(\mathbb{R}^{m}\right)\right)^{k}$ to ensure the existence of the local solution, and we denote the maximal existence time of the classical solution of (4.1) by $T\left(\mathbf{u}_{0}\right)$.

We shall prove the following theorem which describes the behavior of solutions at space infinity. A similar lemma can also be found in 32. to analyze the blow-up problem for a cooperative parabolic system.

Let us introduce several definitions. First we shall define

$$
\|\mathbf{w}(\cdot)\|_{L^{\infty}\left(\mathbb{R}^{m}\right)}:=\sum_{j=1}^{k}\left\|w_{j}(\cdot)\right\|_{L^{\infty}\left(\mathbb{R}^{m}\right)}
$$

for $\mathbf{w}=\left(w_{1}, \ldots, w_{k}\right) \in\left(L^{\infty}\left(\mathbb{R}^{m}\right)\right)^{k}$. We express $\mathbf{u}=\left(u_{1}, \ldots, u_{k}\right) \leq \mathbf{v}=\left(v_{1}, \ldots, v_{k}\right)$ if $u_{j} \leq v_{j}$ for $j=1,2, \ldots, k$ whenever the functions $\mathbf{u}, \mathbf{v}$ are defined. We also write $|\mathbf{u}|:=\max _{j}\left|u_{j}\right|$ for $\mathbf{x}=\left(u_{1}, u_{2}, \ldots, u_{k}\right) \in \mathbb{R}^{k}$. 
Theorem 4.1. Let $\mathbf{u}$ and $\hat{\mathbf{u}}$ be solutions of (4.1) with initial data $\mathbf{u}_{0}, \hat{\mathbf{u}}_{0} \in\left(L^{\infty}\left(\mathbb{R}^{m}\right)\right.$ $\left.\cap C\left(\mathbb{R}^{m}\right)\right)^{k}$, respectively. Suppose that there exist sequences $\left\{r_{n}\right\}_{n=1}^{\infty} \subset(0, \infty)$ and $\left\{a_{n}\right\}_{n=1}^{\infty} \subset \mathbb{R}^{m}$ with $r_{n} \rightarrow \infty$ as $n \rightarrow \infty$ such that

$$
\limsup _{n \rightarrow \infty}\left\|\mathbf{u}_{0}-\hat{\mathbf{u}}_{0}\right\|_{L^{\infty}\left(B_{2 r_{n}}\left(a_{n}\right)\right)}=0 .
$$

Then

$$
\limsup _{n \rightarrow \infty}\|\mathbf{u}(\cdot, t)-\hat{\mathbf{u}}(\cdot, t)\|_{L^{\infty}\left(B_{r_{n}}\left(a_{n}\right)\right)}=0
$$

for any $t \in(0, \tilde{T})$, where $\tilde{T}=\min \left\{T\left(\mathbf{u}_{0}\right), T\left(\hat{\mathbf{u}}_{0}\right)\right\}$.

Proof. First, it is easy to see that the function $\mathbf{X}=\mathbf{u}-\hat{\mathbf{u}}$ satisfies

$$
\begin{aligned}
& \mathbf{X}_{t}=D \Delta \mathbf{X}+\mathbf{f}(\mathbf{u})-\mathbf{f}(\hat{\mathbf{u}}), \quad x \in \mathbb{R}^{m}, 0<t \leq \tilde{T}, \\
& \mathbf{X}(x, 0)=\mathbf{u}_{0}-\hat{\mathbf{u}}_{0} .
\end{aligned}
$$

Let $\varepsilon>0$ and $t_{0} \in(0, \tilde{T})$ be given. By the assumption (4.2), there exists $n_{0} \in \mathbb{N}$ such that

$$
\left\|\mathbf{X}_{0}\right\|_{L^{\infty}\left(B_{2 r_{n}}\left(a_{n}\right)\right)} \leq \varepsilon
$$

for all $n \geq n_{0}$. Since the solutions do not blow up on $(0, \tilde{T})$, the functions

$$
\left|\frac{\partial f_{i}}{\partial x_{j}}(\theta \mathbf{u}+(1-\theta) \hat{\mathbf{u}})\right|
$$

are bounded by some constant $K$ on $\left(0, t_{0}\right)$ for any $\theta \in[0,1]$.

To prove this lemma, we construct a suitable supersolution of the problem (4.3). For this, we define a vector-valued function $\mathbf{Y}$ as a solution of the following problem:

$$
\begin{cases}\mathbf{Y}_{t}=D \Delta \mathbf{Y}+K \mathcal{A} \mathbf{Y}, & x \in \mathbb{R}^{m}, 0<t \leq \tilde{T}, \\ \mathbf{Y}(x, 0)=\mathbf{X}_{0}(x), & x \in \mathbb{R}^{m},\end{cases}
$$

where $\mathcal{A}$ is an $m \times m$ matrix given by

$$
\mathcal{A}=(1)_{1 \leq i \leq k, 1 \leq j \leq k} .
$$

Then the solution $\mathbf{Y}$ of the Cauchy problem (4.6) can be expressed as

$$
\begin{aligned}
\mathbf{Y}(x, t) & =\int_{\mathbb{R}^{m}} G(x-y, t) \mathbf{X}_{0}(y) d y \\
& =\int_{\mathbb{R}^{m} \backslash B_{r_{n}}(x)} G(x-y, t) \mathbf{X}_{0}(y) d y+\int_{B_{r_{n}}(x)} G(x-y, t) \mathbf{X}_{0}(y) d y,
\end{aligned}
$$

where $G=\left(G_{i, j}\right)_{1 \leq i, j \leq k}$ is the Green matrix of the system (4.6).

We shall now use the following estimate (cf. [3]):

$$
\left|\left(\frac{\partial}{\partial x}\right)^{l} G_{i, j}(x, t)\right| \leq C_{l} t^{-\frac{m+|l|}{2}} e^{-C|x|^{2} / t}, \quad 1 \leq i, j \leq k
$$

for all $x \in \mathbb{R}^{m}$ and $t \in\left(0, t_{0}\right)$ for some positive constants $C_{l}, C$, where $l$ is a multiindex. Then the assumption $r_{n} \rightarrow \infty$ as $n \rightarrow \infty$ and the above estimate imply that

$$
G_{i, j}(x-y, t) \leq \int_{|x-y|>r_{n}} C_{0} t^{-\frac{m}{2}} e^{-C|x-y|^{2} / t} d y \leq \frac{\varepsilon}{\beta}
$$


for any large $n \in \mathbb{N}$, where $\beta:=\left\|\mathbf{X}_{0}\right\|_{L^{\infty}\left(\mathbb{R}^{m}\right)}$. From this we can deduce that

$$
\left|\int_{\mathbb{R}^{m} \backslash B_{r_{n}}(x)} G(x-y, t) \mathbf{X}_{0}(y) d y\right| \leq \beta \int_{|x-y|>r_{n}} C_{0} t^{-\frac{m}{2}} e^{-C|x-y|^{2} / t} d y \leq \varepsilon
$$

for any $n$ large.

On the other hand, we note that $x \in B_{r_{n}}\left(a_{n}\right)$ and $y \in B_{r_{n}}(x)$ imply $y \in$ $B_{2 r_{n}}\left(a_{n}\right)$. Thus, from (4.5), there exists a constant $c>0$ such that

$$
\left|\int_{B_{r_{n}}(x)} G(x-y, t) \mathbf{X}_{0}(y) d y\right| \leq \varepsilon \max _{1 \leq i \leq k} \sum_{j=1}^{k} \int_{\mathbb{R}^{m}} G_{i, j}(x-y, t) d y \leq c \varepsilon
$$

for any $x \in B_{r_{n}}\left(a_{n}\right), t \in\left(0, t_{0}\right)$ and $n \in \mathbb{N}$. Consequently, $|\mathbf{Y}(x, t)| \leq(1+c) \varepsilon$ for any $x \in B_{r_{n}}\left(a_{n}\right), t \in\left(0, t_{0}\right)$ and $n \in \mathbb{N}$. By the comparison principle, we have $-\mathbf{Y} \leq \mathbf{X} \leq \mathbf{Y}$, so that

$$
\limsup _{n \rightarrow \infty}\|\mathbf{X}(\cdot, t)\|_{L^{\infty}\left(B_{r_{n}}\left(a_{n}\right)\right)} \leq \limsup _{n \rightarrow \infty}\|\mathbf{Y}(\cdot, t)\|_{L^{\infty}\left(B_{r_{n}}\left(a_{n}\right)\right)}=0, \quad t \in\left(0, t_{0}\right) .
$$

Letting $t_{0}$ vary over $(0, \tilde{T})$, the lemma follows.

This lemma immediately implies the following corollary.

Corollary 4.2. If some solutions of

$$
\mathbf{U}_{t}=\mathbf{f}(\mathbf{U})
$$

blow up in a finite time, then there are spatially inhomogeneous solutions of (4.1) which blow up in a finite time.

Proof. By assumption, there are initial data $\mathbf{U}_{0}$ such that the solution of (4.7) starting from $\mathbf{U}_{0}$ blows up at $t=T\left(\mathbf{U}_{0}\right)<\infty$. Let $\mathbf{u}_{0}$ be spatially inhomogeneous initial data such that

$$
\mathbf{u}_{0} \in\left(L^{\infty}\left(\mathbb{R}^{m}\right) \cap C\left(\mathbb{R}^{m}\right)\right)^{k}, \quad \lim _{|x| \rightarrow \infty} \mathbf{u}_{0}(x)=\mathbf{U}_{0} .
$$

Then Theorem 4.1 implies that the solution of (4.1) starting from $\mathbf{u}(x, 0)=\mathbf{u}_{0}$ blows up in a finite time. Since the solution $\mathbf{u}$ is also spatially inhomogeneous, the proof is complete.

This corollary is applicable to our system (1.2). In the following subsections, we investigate in more detail the behavior of solutions.

Remark 4.3. Fila, Ninomiya and Vázquez [5] constructed the two-component system which exhibits the diffusion-eliminating blow-up. Namely, some solutions of (4.7) blow up in a finite time, while all solutions of (4.1) in a bounded domain with the homogeneous boundary condition converge to $(0,0)$ as $t$ tends to infinity. This corollary also indicates that the boundedness of the domain or the restriction of the solutions at $|x| \rightarrow \infty$ is required for diffusion-eliminating blow-up.

4.2. Global existence. Hereafter, we shall focus on the Cauchy problem for (1.2) such that the initial data satisfy (1.8) and (1.9).

Let $(U(t), V(t))$ be the solution of (1.5) with the initial condition $(U(0), V(0))=$ $(M, N)$. Recall that the solution can be written explicitly as (1.6) with

$$
T_{R}=\frac{M}{M^{2}+N^{2}}, \quad T_{I}=\frac{N}{M^{2}+N^{2}} .
$$

When $N>0$, we can prove Theorem 1.4 by using Theorems 1.1 and 4.1 
Proof of Theorem 1.4. First, we have the local existence of $(u, v)$ for $t \in[0, \tau]$ for some $\tau>0$. By Theorem 4.1 and the explicit form of $(U(t), V(t))$ in (1.6), the assumption of Theorem 1.1 is satisfied for all $|x| \geq R$ at $t=\tau$ for some constants $R \gg 1$ and $A>0$. By the strong maximum principle, we have $v>0$ and $u$ is bounded in $\mathbb{R}^{m} \times[0, \tau]$. This implies that the assumption of Theorem 1.1 holds for all $x$ with $|x| \leq R$ at $t=\tau$ with the constant $A$ (taking a larger one if necessary). Hence, by Theorem 1.1 for the Cauchy problem starting at $t=\tau$, the solution $(u, v)$ is global and converges to $(0,0)$ as $t \rightarrow \infty$. This completes the proof.

4.3. Upper bounds. In this subsection we consider the case $N=0$. Since $V(t) \equiv$ 0 in this case, the equation for $U$ becomes $U_{t}=U^{2}$. We denote the solution of this single equation with $U(0)=M$ by $U_{0}(t):=U_{M, 0}(t)=1 /\left(M^{-1}-t\right)$. The blow-up time of this solution is $T(M):=M^{-1}$.

In order to estimate $u(x, t)$ and $v(x, t)$ from above, we consider the following cooperative system:

$$
\begin{cases}\bar{u}_{t}=\Delta \bar{u}+\bar{u}^{2}, & x \in \mathbb{R}^{m}, t>0, \\ \bar{v}_{t}=\Delta \bar{v}+2 \bar{u} \bar{v}, & x \in \mathbb{R}^{m}, t>0, \\ \bar{u}(x, 0)=\bar{u}_{0}(x), & x \in \mathbb{R}^{m}, \\ \bar{v}(x, 0)=\bar{v}_{0}(x), & x \in \mathbb{R}^{m} .\end{cases}
$$

Also, we consider the following kinetic system corresponding to the problem (4.8):

$$
\begin{cases}\bar{U}_{t}=\bar{U}^{2}, & \bar{U}(0)=M, \\ \bar{V}_{t}=2 \bar{U} \bar{V}, & \bar{V}(0)=L\end{cases}
$$

for some $M, L>0$. We note that the solution of (4.9) is given by

$$
\bar{U}(t)=\frac{1}{T-t}, \quad \bar{V}(t)=\frac{L}{M^{2}} \frac{1}{(T-t)^{2}}, \quad T=T(M):=\frac{1}{M} .
$$

The proof of the following lemma is based on Lemma 2.3 of [18 for the Fujita equation. See also 30] for a quasilinear parabolic equation and 32] for a cooperative parabolic system.

Lemma 4.4. Let $(\bar{U}, \bar{V})$ be the solution of (4.9) and let $(\bar{u}, \bar{v})$ be a solution of (4.8) defined on $\mathbb{R}^{m} \times[0, T)$ with $T:=M^{-1}$. Suppose that there exist $t_{0} \in[0, T)$, $r_{0} \in(0, \infty)$ and a constant $\theta \in(0,1)$ such that

$$
0 \leq \bar{u}(x, t) \leq \theta \bar{U}(t), \quad 0 \leq \bar{v}(x, t) \leq \theta \bar{V}(t) \quad \text { for } \quad|x| \leq r_{0}, t_{0} \leq t<T .
$$

Then $\bar{u}$ and $\bar{v}$ are uniformly bounded in $\left\{|x| \leq r_{0} / 2\right\} \times\left[t_{0}, T\right)$.

Proof. We construct some suitable supersolutions as follows:

$$
w(x, t):=\frac{\hat{\theta}}{T-t+h(r)}, \quad \tilde{w}(x, t):=\frac{L}{M^{2}} \frac{\hat{\theta}}{[T-t+h(r)]^{2}}, \quad r:=|x|,
$$

where $\hat{\theta} \in(\theta, 1)$ and

$$
h(r):=\varepsilon \cos ^{2}\left(\frac{\pi r}{2 r_{0}}\right)
$$


with small $\varepsilon>0$ to be chosen later. We compute

$$
\begin{aligned}
w_{t}-\Delta w-w^{2} & =\frac{w^{2}}{\hat{\theta}}\left\{1-\hat{\theta}+\Delta h-\frac{2|\nabla h|^{2}}{T-t+h}\right\} \\
& \geq \frac{w^{2}}{\hat{\theta}}\left\{1-\hat{\theta}+h_{r r}+\frac{m-1}{r} h_{r}-2 \frac{h_{r}^{2}}{h}\right\} .
\end{aligned}
$$

Since, by the choice of $h$, we have $h_{r r}, h_{r} / r$ and $h_{r}^{2} / h$ are of order $\varepsilon$ for $|r| \leq r_{0}$, we have

$$
\begin{array}{ll}
w_{t} \geq \Delta w+w^{2}, & |x|<r_{0}, t_{0} \leq t<T, \\
w\left(x, t_{0}\right) \geq \bar{u}\left(x, t_{0}\right), & |x| \leq r_{0}, \\
w(x, t) \geq \bar{u}(x, t), & |x|=r_{0}, t_{0} \leq t<T,
\end{array}
$$

if we choose $\varepsilon>0$ sufficiently small. Note that $\varepsilon>0$ is chosen so that

$$
\varepsilon \leq\left(T-t_{0}\right)\left(\frac{\hat{\theta}}{\theta}-1\right)
$$

by $\hat{\theta} \in(\theta, 1)$. By the comparison principle, we have $\bar{u}(x, t) \leq w(x, t)$ for $|x| \leq r_{0}$ and $t_{0} \leq t<T$. Thus we obtain

$$
\bar{u}(x, t) \leq \frac{\hat{\theta}}{T-t+h\left(r_{0} / 2\right)}=\frac{\hat{\theta}}{T-t+\varepsilon / 2} \leq \frac{2 \hat{\theta}}{\varepsilon}<\infty
$$

for any $|x| \leq r_{0} / 2, t_{0} \leq t<T$. Applying a similar argument to $\bar{v}$ and $\tilde{w}$, we complete the proof.

In the following, we assume

$$
\begin{aligned}
& \bar{u}_{0}, \bar{v}_{0} \in C^{1}\left(\mathbb{R}^{m}\right), \quad 0 \leq \bar{u}_{0} \leq M, \quad \bar{u}_{0} \not \equiv M, \quad 0 \leq \bar{v}_{0} \leq L, \quad \bar{v}_{0} \not \equiv L, \\
& \lim _{|x| \rightarrow \infty} \bar{u}_{0}(x)=M, \lim _{|x| \rightarrow \infty} \bar{v}_{0}(x)=L
\end{aligned}
$$

for some constants $M>0$ and $L>0$. The following lemma shows that blow-up can occur only at space infinity.

Lemma 4.5. Let $(\bar{u}, \bar{v})$ be a solution of (4.8) satisfying (4.10) and (4.11). Then $\bar{u}$ and $\bar{v}$ blow up at the finite time $t=T(M):=M^{-1}$. Moreover, $\bar{u}$ and $\bar{v}$ are uniformly bounded in $\mathcal{K} \times[0, T)$ for any compact set $\mathcal{K} \subset \mathbb{R}^{m}$.

Proof. By the comparison principle, we have $0 \leq \bar{u}(x, t) \leq \bar{U}(t)$ and $0 \leq \bar{v}(x, t) \leq$ $\bar{V}(t)$ for all $x \in \mathbb{R}^{m}$ and $t>0$. On the other hand, applying Theorem 4.1 with $\left|a_{n}\right|=4 n$ and $r_{n}=n$, we obtain

$$
\lim _{|x| \rightarrow \infty} \bar{u}(x, t)=\bar{U}(t), \quad \lim _{|x| \rightarrow \infty} \bar{v}(x, t)=\bar{V}(t), \quad t \in[0, T(M)) .
$$

Combining the above two facts, we obtain the blow-up time $T\left(\bar{u}_{0}, \bar{v}_{0}\right)=T(M)=$ $1 / M$.

It remains to prove the uniform boundedness of $\bar{u}$ and $\bar{v}$ in $\mathcal{K} \times[0, T)$ for any given compact subset $\mathcal{K}$ of $\mathbb{R}^{m}$. For this purpose, in view of Lemma 4.4 it suffices to show that, for any given $R>0$ there exist $t_{0} \in[0, T)$ and $\theta \in(0,1)$ such that

$$
0 \leq \bar{u}(x, t) \leq \frac{\theta}{T-t}, \quad 0 \leq \bar{v}(x, t) \leq \frac{L}{M^{2}} \frac{\theta}{(T-t)^{2}}, \quad|x| \leq 2 R, t_{0} \leq t<T .
$$


For this, we let $\gamma(x, t):=\bar{u}(x, t) / \bar{U}(t)$. Then the function $\gamma=\gamma(x, t)$ satisfies

$$
\gamma_{t}=\Delta \gamma+\bar{U}\left(\gamma^{2}-\gamma\right) \leq \Delta \gamma
$$

since $0 \leq \gamma \leq 1$. Moreover, by (4.10) and (4.11) we have

$$
0 \leq \gamma(\cdot, 0)=\frac{\bar{u}_{0}}{M} \leq 1, \quad \gamma(\cdot, 0) \not \equiv 1 .
$$

From the strong maximum principle, we see that $0 \leq \gamma(x, t)<1$ for all $x \in \mathbb{R}^{m}$ and $t>0$. Therefore, for any $R>0$ there exist $\theta \in(0,1)$ and $t_{0} \in(0, T)$ such that

$$
0 \leq \gamma(x, t) \leq \theta, \quad|x| \leq 2 R, t_{0} \leq t<T .
$$

This gives the first inequality in (4.12). Next, we define $\chi(x, t):=\bar{v}(x, t) / \bar{V}(t)$. Arguing as above, this function $\chi=\chi(x, t)$ satisfies

$$
\chi_{t}=\Delta \chi+2 \bar{U} \chi(\gamma-1) \leq \Delta \chi, \quad 0 \leq \chi(\cdot, 0) \leq 1 \quad \text { and } \quad \chi(\cdot, 0) \not \equiv 1 .
$$

The second inequality of (4.12) can be proved similarly. Thus the proof is completed.

4.4. Nonsimultaneous blow-up. Now we are in a position to complete the proof of Theorem 1.5 .

Proof of Theorem 1.5. First, we choose functions $\bar{u}_{0}, \bar{v}_{0}$ as $\bar{u}_{0}=u_{0}$ and $\bar{v}_{0} \geq v_{0}$ such that $\bar{v}_{0}$ satisfies conditions (4.10) and (4.11) with the same constants $M, L$ as in (1.8) and (1.9). Then, by the comparison principle, we have

$$
u(x, t) \leq \bar{u}(x, t), 0 \leq v(x, t) \leq \bar{v}(x, t), \quad x \in \mathbb{R}^{m}, t>0 .
$$

By Lemma 4.5, the solution $(\bar{u}, \bar{v})$ blows up simultaneously at time $t=T(M)=$ $1 / M$ only at space infinity. Thus the inequality (4.13) implies that the component $v$ does not blow up until the time $t=T(M)$ and $\lim _{t \rightarrow T(M)} v(x, t)<\infty$ for all fixed $x \in \mathbb{R}^{m}$. Furthermore, by using Theorem 4.1 with $\left|a_{n}\right|=4 n$ and $r_{n}=n$, we obtain

$$
\lim _{|x| \rightarrow \infty} u(x, t)=U_{0}(t), \quad \lim _{|x| \rightarrow \infty} v(x, t)=0, \quad t \in[0, T(M)) .
$$

Thus $\lim _{t \rightarrow T(M)}\|v(\cdot, t)\|_{L^{\infty}\left(\mathbb{R}^{m}\right)}$ is bounded.

The boundedness of $v$ implies that the component $u$ is bounded below up to the time $t=T(M)$. Hence there exists a constant $C>0$ such that

$$
-C<u \leq \bar{u} \text { in } \mathbb{R}^{m} \times[0, T(M)) .
$$

These inequalities and (4.14) imply that $\|u(\cdot, t)\|_{L^{\infty}\left(\mathbb{R}^{m}\right)} \rightarrow \infty$ as $t \nearrow T(M)$. Hence we obtain $T\left(u_{0}, v_{0}\right)=T(M)$. On the other hand, from Lemma 4.5 $\bar{u}$ blows up only at space infinity. Combining this with (4.15), we conclude that the blow-up of the component $u$ occurs only at space infinity.

\section{ACKNOWLEDGEMENTS}

The authors would like to thank Professors Marek Fila and Peter Poláčik for their valuable comments. The first author was partially supported by the National Science Council of the Republic of China under the grants NSC 98-2115-M-003001 and NSC 99-2115-M-032-006-MY3. The second author was partially supported by the Challenging Exploratory Research (No. 23654042). The third author was partially supported by the Grant-in-Aid for Young Scientist (B) (No. 40588779) and the fourth author was partially supported by the Grant-in-Aid for Scientific Research (A) (No. 19204014) from the Japan Society for the Promotion of Science. 


\section{REFERENCES}

[1] K. Deng, Blow-up rates for parabolic systems, Z. Angew. Math. Phys. 47 (1996), 132-143. MR 1408675 (98f:35080)

[2] K. Deng and H. A. Levine, The role of critical exponents in blow-up theorems, J. Math. Anal. Appl. 243 (2000), 85-126. MR 1742850 (2001b:35031)

[3] S. D. Ėŭdel'man, Parabolic Systems, North-Holland Publ. Co. 1969. MR0252806 (40:6023)

[4] M. Escobedo and M. A.Herrero, Boundedness and blow up for a semilinear reaction-diffusion system, J. Differential Equations 89 (1991), 176-202. MR1088342 (91j:35040)

[5] M. Fila, H. Ninomiya and J.L. Vázquez, Dirichlet boundary conditions can prevent blowup reaction-diffusion equations and systems, Discr. Cont. Dyn. Systems 14 (2006), 63-74. MR2170313(2007b:35194)

[6] M. Fila and Ph. Souplet, The blow-up rate for semilinear parabolic problems on general domains. NoDEA Nonlinear Differential Equations Appl. 8 (2001), 4, 473-480. MR.1867324 (2002h:35116)

[7] A. Friedman and Y. Giga, A single point blow-up for solutions of semilinear parabolic systems, J. Fac. Sci. Univ. Tokyo Sect. IA, Math. 34 (1987), 65-79. MR882125 (89b:35066)

[8] A. Friedman and B. McLeod, Blow-up of positive solutions of semilinear heat equations, Indiana Univ. Math. J. 34 (1985), 425-447. MR783924(86j:35089)

[9] H. Fujita, On the blowing up of solutions of the Cauchy problem for $u_{t}=\Delta u+u^{1+\alpha}$. J. Fac. Sci. Univ. Tokyo Sec. IA Math. 13 (1966), 109-124. MR0214914 (35:5761)

[10] Y. Giga and R.V. Kohn, Asymptotically self-similar blow-up of semilinear heat equations, Comm. Pure Appl. Math., 38 (1985), 297-319. MR784476 (86k:35065)

[11] Y. Giga and R. V. Kohn, Characterizing blowup using similarity variables, Indiana Univ. Math. J., 36 (1987), 1-40. MR876989(88c:35021)

[12] Y. Giga and R. V. Kohn, Nondegeneracy of blowup for semilinear heat equations, Comm. Pure Appl. Math., 42 (1989), 845-884. MR1003437 (90k:35034)

[13] Y. Giga and N. Umeda, On blow-up at space infinity for semilinear heat equations, J. Math. Anal. Appli. 316 (2006), no. 2, 538-555. MR2206688(2007b:35175)

[14] J.-S. Guo, S. Sasayama and C.-J. Wang, Blowup rate estimate for a system of semilinear parabolic equations, Comm. Pure Appl, Anal. 8 (2009), 711-718. MR2461571(2009m:35197)

[15] M.A. Herrero and J.J.L. Velazquez, Explosion de solutions d'équations paraboliques semilinéaires supercritiques, C. R. Acad. Sci. Paris Sér. I Math., 319 (1994), 141-145 . MR 1288393 (95i:35037)

[16] O.A. Ladyzenskaja, V.A. Solonnikov and N.N. Ural'ceva, Linear and Quasilinear Equations of Parabolic Type, Transl. Math. Monographs, 23, 1967. MR0241822 (39:3159b)

[17] H. Matano and F. Merle, On nonexistence of type II blowup for a supercritical nonlinear heat equation, Comm. Pure Appl. Math., 57 (2004), 1494-1541. MR2077706 (2005e:35115)

[18] H. Matano and F. Merle, Classification of type I and type II blowup for a supercritical nonlinear heat equation, J. Funct. Anal. 256 (2009), 992-1064. MR2488333 (2009k:35124)

[19] N. Mizoguchi, Rate of type II blowup for a semilinear heat equation, Math. Ann. 339 (2007), 839-877. MR2341904 (2008k:35246)

[20] K. Mochizuki and Q. Huang, Existence and behavior of solutions for a weakly coupled system of reaction-diffusion equations, Methods Appl. Anal. 5 (2) (1998), 109-124. MR.1636542 (99e:35109)

[21] N. Nouaili and H. Zaag, A Liouville theorem for vector valued semilinear heat equations with no gradient structure and applications to blow-up, Trans. Amer. Math. Soc. 362 (2010), no. 7, 3391-3434. MR2601595 (2011f:35184)

[22] H. Okamoto, T. Sakajo and M. Wunsch, On a generalization of the Constantin-Lax-Majda equation, Nonlinearity 21 (2008), no. 10, 2447-2461. MR2439488 (2009g:76009)

[23] R. Palais, Blowup for nonlinear equations using a comparison principle in Fourier space, Comm. Pure Appl. Math., 41 (1988), pp. 165-196. MR924683 (89c:35083)

[24] F. Quiros and J. D. Rossi, Non-simultaneous blow-up in a semilinear parabolic system, Z. angew. Math. Phys. 52 (2001) 342-346 MR1834531 (2002c:35145)

[25] P. Quittner and Ph. Souplet, Superlinear Parabolic Problems. Blow-up, Global Existence and Steady States, Birkhäuser Advanced Texts, Basler Lehrbücher, 2007. MR2346798 (2008f:35001) 
[26] J. D. Rossi and Ph. Souplet, Coexistence of simultaneous and nonsimultaneous blow-up in a semilinear parabolic system, Differential Integral Equations 18 (2005), no. 4, 405-418. MR2122706 (2006b:35151)

[27] T. Sakajo, Blow-up solutions of the Constantin-Lax-Majda equation with a generalized viscosity term, J. Math. Sci. Univ. Tokyo 10 (2003), no. 1, 187-207. MR1963803(2004e:35187)

[28] T. Sakajo, On global solutions for the Constantin-Lax-Majda equation with a generalized viscosity term, Nonlinearity 16 (2003) 1319-1328 MR.1986297(2004d:76029)

[29] S. Schochet, Explicit solutions of the viscous model vorticity equation, Commun. Pure. Appl. Math. 39 (1986) 531-537 MR840339 (87h:35322)

[30] Y. Seki, On directional blow-up for quasilinear parabolic equations with fast diffusion, J. Math. Anal. Appl. 338 (2008), 572-587. MR2386440(2009d:35164)

[31] M. Shimojo, The global profile of blow-up at space infinity in semilinear heat equations, Journal of Mathematics of Kyoto University. 48 (2008), 339-361. MR2436740(2009h:35181)

[32] M. Shimojo and N.Umeda, Blow-up at space infinity for solutions of cooperative reactiondiffusion systems, Funkcialaj Ekvacioj 54 (2011), 315-334. MR2867018

[33] Ph. Souplet, Single point blow-up for a semilinear parabolic system, J. Eur. Math. Soc. 11 (2009), 169-188. MR2471135 (2010b:35205)

[34] Ph. Souplet and S. Tayachi, Optimal condition for non-simultaneous blow-up in a reactiondiffusion system, J. Math. Soc. Japan 56, 2 (2004), 571-584. MR2048475 (2005a:35117)

[35] R. Redheffer and W. Walter, Invariant sets for systems of partial differential equations I. Parabolic equations, Archive Rational Mech. Anal. 67 (1977), 41-52. MR 473317(82h:35050)

[36] M. Wang, Blow-up rate estimates for semilinear parabolic systems, J. Diff. Equations 170 (2001), 317-324. MR1815186 (2001k:35159)

[37] H. Weinberger, Invariant sets for weakly coupled parabolic and elliptic systems, Rend. Mat. 8 (1975), 295-310. MR0397126(53:986)

Department of Mathematics, Tamkang University, Tamsui, Taipei County 25137, TaiWAN

E-mail address: jsguo@mail.tku.edu.tw

Department of Mathematics, Meiji University, 1-1-1 Higashimita, Tamaku, Kawasaki 214-8571, JAPAN

E-mail address: ninomiya@math.meiji.ac.jp

Department of Mathematics, Meiji University, 1-1-1 Higashimita, Tamaku, Kawasaki 214-8571, JAPAN

E-mail address: shimojotw@gmail.com

Department of Mathematics, Tokyo Institute of Technology, Meguro-ku, Tokyo 152-8551, JAPAN

E-mail address: yanagida@math.titech.ac.jp 